\title{
Describable Visual Attributes for Face Verification and Image Search
}

\author{
Neeraj Kumar, Student Member, IEEE, Alexander C. Berg, Member, IEEE, \\ Peter N. Belhumeur, and Shree K. Nayar, Member, IEEE
}

\begin{abstract}
We introduce the use of describable visual attributes for face verification and image search. Describable visual attributes are labels that can be given to an image to describe its appearance. This paper focuses on images of faces and the attributes used to describe them, although the concepts also apply to other domains. Examples of face attributes include gender, age, jaw shape, nose size, etc. The advantages of an attribute-based representation for vision tasks are manifold: they can be composed to create descriptions at various levels of specificity; they are generalizable, as they can be learned once and then applied to recognize new objects or categories without any further training; and they are efficient, possibly requiring exponentially fewer attributes (and training data) than explicitly naming each category. We show how one can create and label large datasets of real-world images to train classifiers which measure the presence, absence, or degree to which an attribute is expressed in images. These classifiers can then automatically label new images. We demonstrate the current effectiveness - and explore the future potential - of using attributes for face verification and image search via human and computational experiments. Finally, we introduce two new face datasets, named FaceTracer and PubFig, with labeled attributes and identities, respectively.
\end{abstract}

Index Terms-Face recognition, attribute classification, classifier training, content-based image retrieval, image search.

\section{INTRODUCTION}

$\mathrm{O}$ NE of history's most successful books was a fivevolume pharmacopoeia titled De Materia Medica, written in the first century by the Greek botanist and physician Pedanius Dioscorides. It is perhaps the earliest known field guide, giving pictures and written descriptions of nearly 600 plant species, showing how each could be found and identified. This work would be the first in a line of botanical texts, including the ninth century medieval agricultural and toxicological texts of Ibn Washiyah, and the early eighteenth century Systema Naturae of Carl Linneaus, which laid out the rules of modern taxonomy. All of these works have in common an effort to teach the reader how to identify a plant or animal by describable aspects of its visual appearance.

While the use of describable visual attributes for identification has been around since antiquity, it has not been the focus of work by researchers in computer vision and related disciplines. Most existing methods for recognition (e.g., [13], [36], [38], [54]) work by extracting low-level features in images, such as pixel values, gradient directions, histograms of oriented gradients [13], SIFT [34], etc., which are then used to directly train classifiers for identification or detection.

In contrast, we use low-level image features to first learn intermediate representations [29], [30], in which

- The authors are with the Computer Science Department, Columbia University, New York, NY 10027.

Email: \{neeraj, belhumeur, nayar\}@cs.columbia.edu and aberg@cs.stonybrook.edu images are labeled with an extensive list of descriptive visual attributes. Although these attributes could clearly be useful in a variety of domains (such as object recognition, species identification, architectural description, action recognition, etc.), we focus solely on faces in this paper. These face attributes can range from simple demographic information such as gender, age, or ethnicity; to physical characteristics of a face such as nose size, mouth shape, or eyebrow thickness; and even to environmental aspects such as lighting conditions, facial expression, or image quality. In our approach, an extensive vocabulary of visual attributes is used to label a large dataset of images, which is then used to train classifiers that automatically recognize the presence, absence, or degree to which these attributes are exhibited in new images. The classifier outputs can then be used to identify faces and search through large image collections, and they also seem promising for use in many other tasks such as image exploration or automatic description-generation.

Why might one need these attributes? What do they afford? Why not train classifiers directly for the task at hand? Visual attributes - much like words - are composable, offering tremendous flexibility and efficiency. Attributes can be combined to produce descriptions at multiple levels, including object categories, objects, or even instances of objects. For example, one can describe "white male" at the category level (a set of people), or "white male brown-hair green-eyes scaron-forehead" at the object level (a specific person), or add "..., smiling lit-from-above seen-from-left" to the previous for an instance of the object (a particular image of a person). 


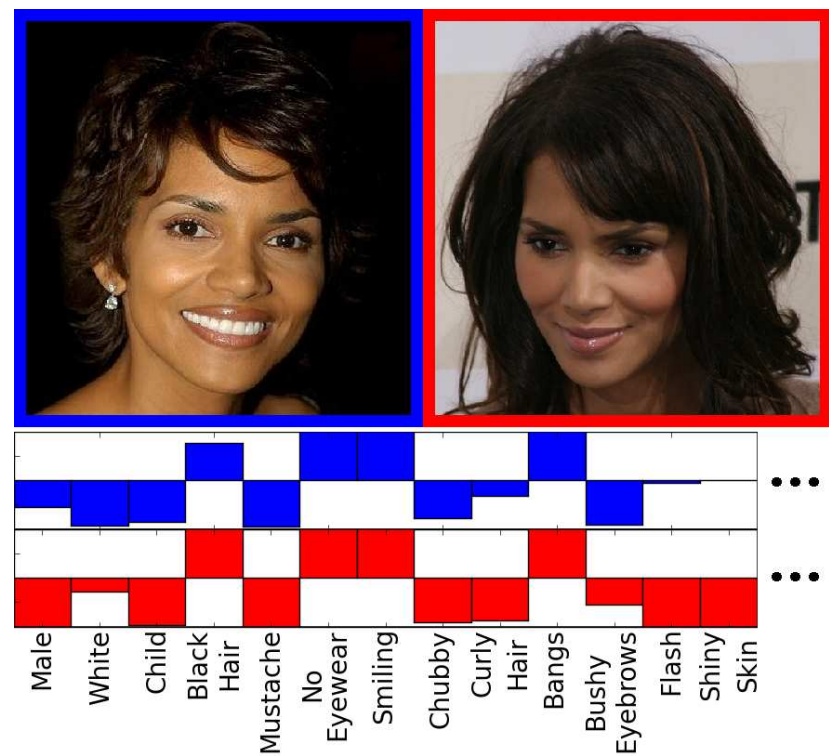

(a) Attribute values for two images of the same person

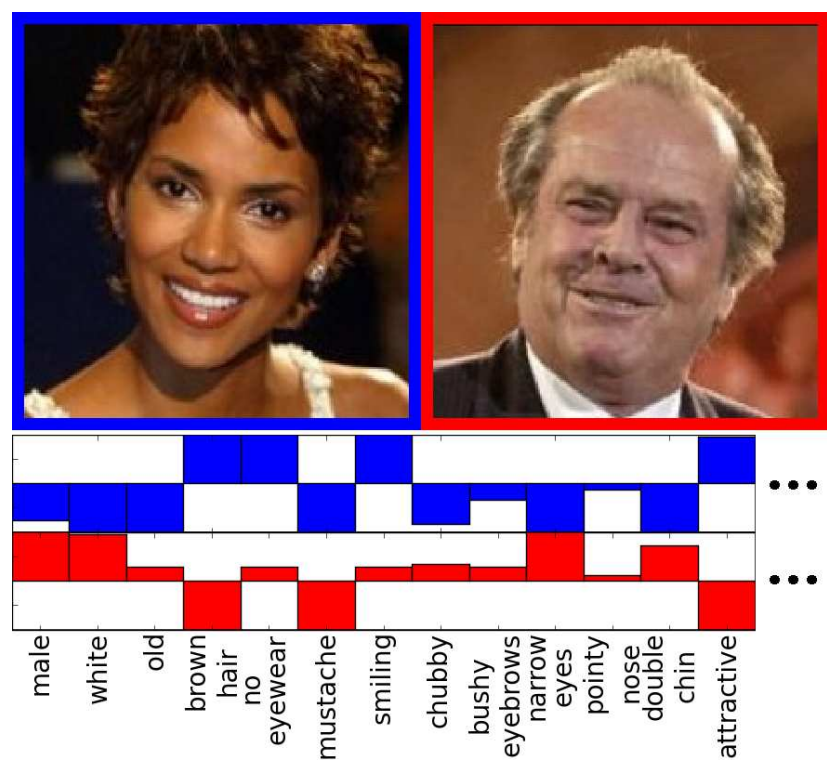

(b) Attribute values for images of two different people

Fig. 1. An attribute classifier can be trained to recognize the presence or absence of a describable visual attribute. The responses for several such attribute classifiers are shown for (a) two images of the same person and (b) two images of different individuals. In (a), notice how most attribute values are in strong agreement, despite the changes in pose, illumination, expression, and image quality. Conversely, in (b), the values differ completely despite the similarity in these same environmental aspects. We train a verification classifier on these outputs to perform face verification, achieving $85.54 \%$ accuracy on the Labeled Faces in the Wild (LFW) benchmark [27], comparable to the state-of-the-art.

Moreover, attributes are generalizable; one can learn a set of attributes from large image collections and then apply them in almost arbitrary combinations to novel images, objects, or categories. Better still, attributes are efficient: consider that $k$ binary attributes may suffice to identify $2^{k}$ categories, clearly more efficient than naming each category individually. (Of course, in practice, the potential benefits are limited by the problem domain, the type of categories being considered, and the accuracy of learned classifiers.) In contrast to existing labeling efforts such as ImageNet [15] and LabelMe [48] that label large collections of images by category or object name, the use of attributes may provide a significantly more compact way of describing objects. This would allow for the use of much smaller labeled datasets to achieve comparable performance on recognition tasks.

Perhaps most importantly, these attributes can be chosen to align with the domain-appropriate vocabulary that people have developed over time for describing different types of objects. For faces, this includes descriptions at the coarsest level (such as gender and age) to more subtle aspects (such as expressions and shape of face parts) to highly face-specific marks (such as moles and scars).

While describable visual attributes are one of the most natural ways of describing faces, a person's appearance can also be described in terms of the similarity of a part of their face to the same part of another individual's. For example, someone's mouth might be like Angelina Jolie's, or their nose like Brad
Pitt's. Dissimilarities also provide useful information - e.g., her eyes are not like Jennifer Aniston's. We call these "similes."

In this work, we show two major uses of classifiers trained on describable visual attributes and similes: face verification and image search. Face verification is the problem of determining whether two faces are of the same individual. What makes this problem difficult is the enormous variability in the manner in which an individual's face presents itself to a camera: not only might the pose differ, but so might the expression and hairstyle. Making matters worse - at least for researchers in biometrics - is that the illumination direction, camera type, focus, resolution, and image compression are all almost certain to vary as well. These manifold differences in images of the same person have confounded methods for automatic face recognition and verification, often limiting the reliability of automatic algorithms to the domain of more controlled settings with cooperative subjects [4], [22], [24], [44], [46], [49], [51].

We approach the unconstrained face verification problem (with non-cooperative subjects) by comparing faces using our attribute and simile classifier outputs, instead of low-level features directly. Fig. 1 shows the outputs of various attribute classifiers, for (a) two images of the same person and (b) images of two different people. Note that in (a), most attribute values are in strong agreement, despite the changes in pose, illumination, and expression, while in (b), the values are almost perfectly contrasting. By training 


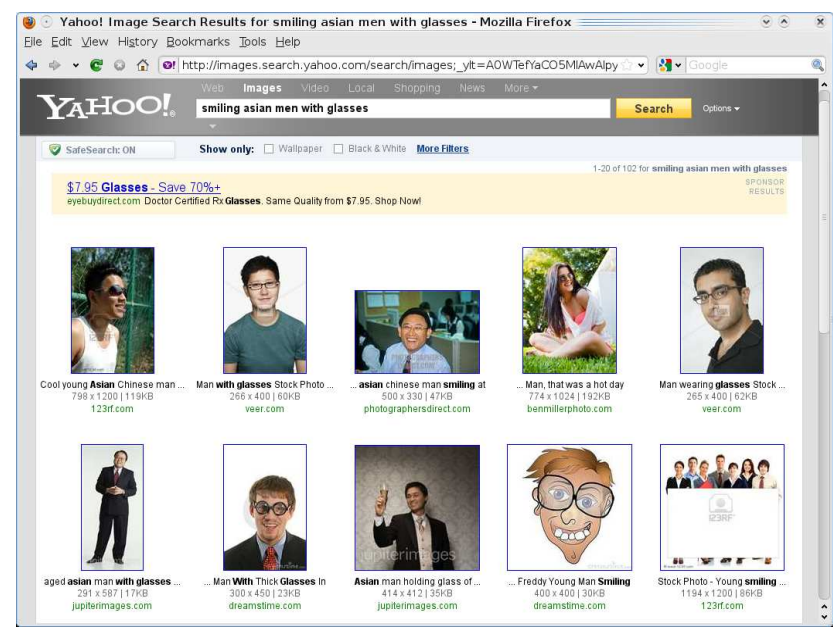

(a) Yahoo image search results

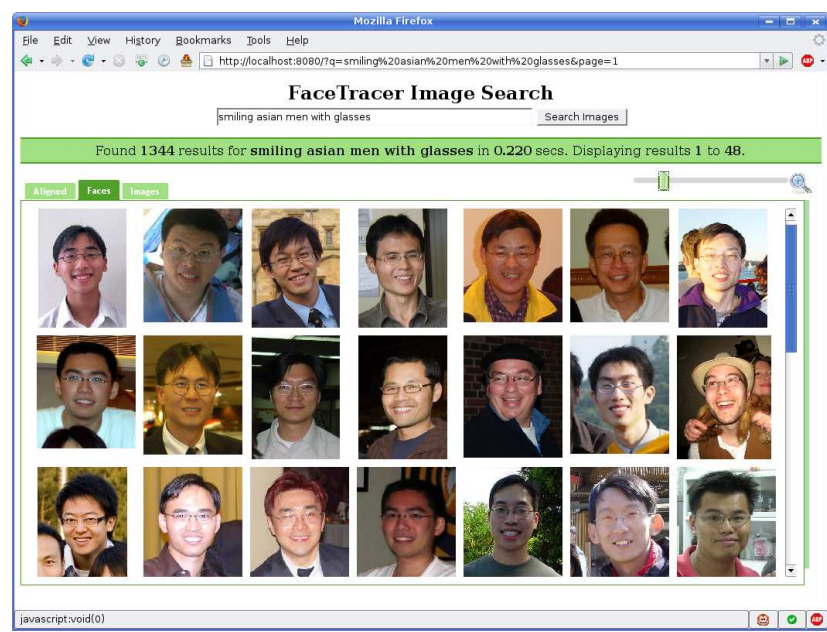

(b) Attribute-based image search results

Fig. 2. Results for the query, "smiling asian men with glasses," using (a) the Yahoo image search engine (as of November 2010) and (b) our face search engine. Conventional image search engines rely on text annotations, such as file metadata, manual labels, or surrounding text, which are often incorrect, ambiguous, or missing. In contrast, we use attribute classifiers to automatically label images with faces in them, and store these labels in a database. At search time, only this database needs to be queried, and results are returned instantaneously. The attribute-based search results are much more relevant to the query.

a classifier that uses these labels as inputs for face verification, we achieve close to state-of-the-art performance on the Labeled Faces in the Wild (LFW) data set [27], at $85.54 \%$ accuracy.

LFW is remarkable for its variability in all of the aspects of visual appearance mentioned above, which also makes it a challenging benchmark for face verification algorithms. Our excellent performance on this benchmark shows that our particular approach to building and using attribute classifiers is, at the very least, adequate; however, how much better could one do? The attribute classifiers we train are currently binary, with continuous outputs approximated by the distance of a sample to the classification boundary. One could instead train regressors to directly estimate real-valued attribute outputs with greater accuracy. An upper-bound on the expected accuracy of attribute classification can be found by asking humans to label attributes. Thus, replacing the automatic classifier outputs with human labels, we found that accuracy on LFW goes up to $91.86 \%$. Going even further, we asked humans to do the entire verification process. This experiment revealed the ideal to which automatic algorithms should aspire $-99.20 \%$.

Given the tremendous strides in face recognition performance over the last two decades, in large part due to the introduction of larger and more realistic data sets, we have publicly released two large datasets: FaceTracer, which contains URLs to 15,000 face images and 5,000 attribute labels; and PubFig, which contains URLs to 58,797 images of 200 public figures - politicians and celebrities.

Another application of describable visual attributes is image search. The ability of current search engines to find images based on facial appearance is limited to images with text annotations. Yet, there are many problems with annotation-based image search: the manual labeling of images is time-consuming; the annotations are often incorrect or misleading, as they may refer to other content on a webpage; and finally, the vast majority of images are simply not annotated. Figs. $2 \mathrm{a}$ and $2 \mathrm{~b}$ show the results of the query, "smiling asian men with glasses," using a conventional image search engine (Yahoo Image Search, as of November 2010) and our search engine, respectively. The difference in quality of search results is clearly visible. Yahoo's reliance on text annotations causes it to find some images that have no relevance to the query, while our system returns only the images that match the query. In addition, many of the correct results on Yahoo point to stock photography websites, which can afford to manually label their images with keywords - but only because they have collections of a limited size, and they label only the coarsest attributes. Clearly, this approach does not scale.

Both of our systems first require the creation of a large dataset of real-world face images. This is done by downloading images from the internet, running face detection and alignment, and then obtaining ground-truth attribute labels, all of which is described in Sec. 3. From this labeled data, one can train accurate attribute and simile classifiers fully automatically, as described in Sec. 4. Performing face verification using these attributes is described in Sec. 5, which also contains various experiments and looks at how well people perform on verification. Finally, the use of attributes for searching in large collections of images with faces is described in Sec. 6 . 


\section{Related Work}

Our work lies at the intersection of attribute classification, face verification and content-based image retrieval. We present an overview of the relevant work, organized by these topics.

\subsection{Attribute Classification}

Prior research on attribute classification has focused mostly on gender and ethnicity classification. Early works [12], [23] used neural networks to perform gender classification on small datasets. The Fisherfaces work [2] showed that linear discriminant analysis could be used for simple attribute classification such as glasses/no glasses. Later, Moghaddam and Yang [35] used Support Vector Machines (SVMs) [11] trained on small "face-prints" to classify the gender of a face, showing good results on the FERET face database [44]. The works of Shakhnarovich et al. [50] and Baluja and Rowley [1] used Adaboost [21] to select a linear combination of weak classifiers, allowing for almost real-time classification of face attributes, with results in the latter case again demonstrated on the FERET database. These methods differ in their choice of weak classifiers: the former uses the Haarlike features of the Viola-Jones face detector [57], while the latter uses simple pixel comparison operators. In a more general setting, Ferrari and Zisserman [20] described a probabalistic approach for learning simple attributes such as colors and stripes.

In computer vision, the use of attributes has recently been receiving much attention from a number of different groups. This journal paper builds on earlier conference works [29], [30]. Other contemporaneous works that use attributes to describe objects include [31], for animal categorization, and [18], for building general attribute predictors. However, the focus of all of these papers is quite different. The latter [18] explores how to train attribute classifiers in a very general setting (such as for evaluation on the Pascal VOC challenge [17]) and the problems associated with, e.g., correlations in training data. The former [31], on the other hand, focuses on trying to distinguish animal species and transfer labels across categories. In contrast to both of these approaches, which are trying to find relations across different categories, we concentrate on finding relations between objects in a single category: faces.

Faces have many advantages compared to generic object categories. There is a well-established and consistent reference frame to use for aligning images; differentiating objects is conceptually simple (e.g., it's unclear whether two cars of the same model should be considered the same object or not, whereas no such difficulty exists for two faces); and most attributes can be shared across all people (unlike, e.g., "4-legged," "gothic," or "dual-exhaust," which are applicable to animals, architecture, and automobiles, respectively
- but not to each other). All of these benefits make it possible for us to train more reliable and useful classifiers, and demonstrate results comparable to the state-of-the-art.

In psychology and neuroscience, there have been a number of works on face recognition as done by humans. The work of Bruce et al. [5] addresses many aspects of human recognition of faces in video and images, including results showing that people are very robust to decreased resolution when recognizing familiar faces, and that the face itself is more useful than the body or gait in such settings [6]. In contrast, Sinha and Poggio [53] show an example where context dominates image information in the face region itself. In later work, Sinha et al. [52] provide a wide-ranging overview of results from psychology on face recognition, briefly covering the work of Bruce et al. [5] and also discussing the effects of varying many other imaging conditions.

Exciting recent work [41] considers explicitly training attribute classifiers for words, in order to decode fMRI measurements of brain activity while subjects think about words. This work includes initial PACstyle bounds on attribute-based zero-shot learning.

\subsection{Face Verification}

Early work in appearance-based face verification [28], [56] looked at the $L_{2}$ distance between pairs of images in a lower dimensional subspace obtained using Principal Components Analysis (PCA). This was extended and improved upon by using linear discriminant analysis [2]. However, these algorithms are mostly limited to images taken in highly controlled environments with extremely cooperative subjects. It is well understood that variation in pose and expression and, to a lesser extent, lighting cause significant difficulties for recognizing the identity of a person [61]. Illumination changes can be mostly handled using a variety of different approaches; the direction of the image gradient [9] and related image features such as SIFT [34], the phase of Gabor jets [58], and gradient pyramids [33] are all highly insensitive to lighting variation. The CMU Pose, Illumination, and Expression (PIE) data set and follow-on results showed that sometimes alignment, especially in $3 \mathrm{D}$, can overcome the other difficulties [4], [7], [10], [24], [51].

Unfortunately, in the setting of real-world images such as those in the "Labeled Faces in the Wild" (LFW) benchmark data set [27] and similar data sets [3], [16], 3D alignment is difficult and has not (yet) been successfully demonstrated. Various 2D alignment strategies have been applied to LFW - aligning all faces [25] to each other, or aligning each pair of images to be considered for verification [19], [37]. Approaches that require alignment between each image pair are computationally expensive. Our work does not require pairwise alignment. Neither do many 


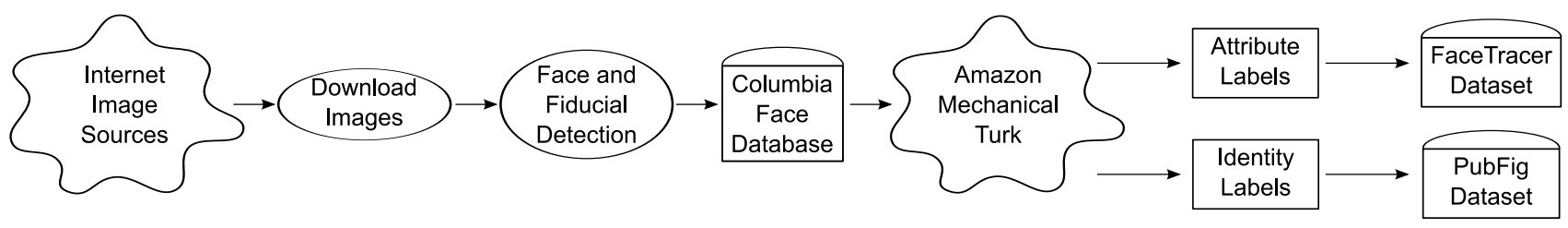

Fig. 3. Creating labeled image datasets: Our system downloads images from the internet. These images span many sources of variability, including pose, illumination, expression, cameras, and environment. Next, faces and fiducial points are detected using a commercial detector [39] and stored in the Columbia Face Database. A subset of these faces are submitted to the Amazon Mechanical Turk service, where they are labeled with attributes or identity, which are used to create the FaceTracer and PubFig datasets, respectively. Both datasets have been publicly released for non-commercial use.

other recent methods on LFW [47], [55], [59], [60], all of which use a large set of carefully designed local features. The best-performing of these [60] ranks the similarity of each face in an input pair to those in a "background set," which is similar in spirit to our simile classifiers.

\subsection{Content-Based Image Retrieval (CBIR)}

Our search application can be viewed as a form of CBIR, where our content is limited to images with faces. Interested readers can refer to the work of Datta et al. [14] and Lew et al. [32] for a recent survey of this field. Most relevant to our work is the "Photobook" system [43], which allows for similaritybased searches of faces and objects using parametric eigenspaces. However, their goal is different from ours. Whereas they try to find objects similar to a chosen one, we locate a set of images starting only with simple text queries. Although we use vastly different classifiers and methods for feature selection, their division of the face into functional parts such as the eyes, nose, etc., is echoed in our approach of training classifiers on functional face regions. While in this paper we ignore existing text annotations for images, one could envision using describable attributes in combination with such annotations for improved search performance, somewhat akin to the idea presented in the "Names and Faces" work [3].

\section{Creating Labeled Image Datasets}

Two recent trends in internet services have made collecting and labeling image data dramatically easier. First, large internet photo-sharing sites such as flickr.com and picasa.com are growing exponentially and host billions of public images, some with textual annotations and comments. In addition, search engines such as Google Images allow searching for images of particular people (albeit not perfectly). Second, efficient marketplaces for online labor, such as Amazon's Mechanical Turk (MTurk) ${ }^{1}$, make it possible to label thousands of images easily and with very low overhead. We exploit both of these trends to create a large dataset of real-world images with attribute and identity labels, as shown in Fig. 3 and described next.

1. http://mturk.com

\subsection{Collecting Face Images}

We use a variety of online sources for collecting face images, including search engines such as Yahoo Images and photo-sharing websites such as flickr.com. Depending on the type of data needed, one can either search for particular people's names (to build a dataset labeled by identity) or for default image filenames assigned by digital cameras (to use for labeling with attributes). The latter technique allows one to find images that are otherwise not returned in most users' queries, i.e., images which are effectively "invisible." Relevant metadata such as image and page URLs are stored in the EXIF tags of the downloaded images.

Next, we apply the OKAO face detector [39] to the downloaded images to extract faces. This detector also returns the pose angles of each face, as well as the locations of six fiducial points: the corners of both eyes and the corners of the mouth. These fiducial points are used to align faces to a canonical pose, via an affine transformation computed using linear least squares on the detected points and corresponding points defined on a template face. The 3.1 million aligned faces collected using this procedure comprise the Columbia Face Database.

We make two observations about this database. First, from the statistics of the randomly-named images, it appears that a significant fraction of them contain faces $(25.7 \%)$, and on average, each image contains 0.5 faces. Thus, it is clear that faces are ubiquitous and an image case to understand. Second, our collection of aligned faces is the largest such collection of which we are aware. It is truly a "realworld" dataset, with completely uncontrolled lighting and environments, taken using unknown cameras and in unknown imaging conditions, with a wide range of image resolutions. In contrast, existing face datasets such as Yale Face A\&B [22], CMU PIE [51], and FERET [44] are either much smaller in size and/or taken in highly controlled settings. Even the more expansive FRGC version 2.0 dataset [45] has a limited number of subjects, image acquisition locations, and all images were taken with the same camera type. The most comparable dataset is LFW [27], itself derived from earlier work [3]. These images were collected 


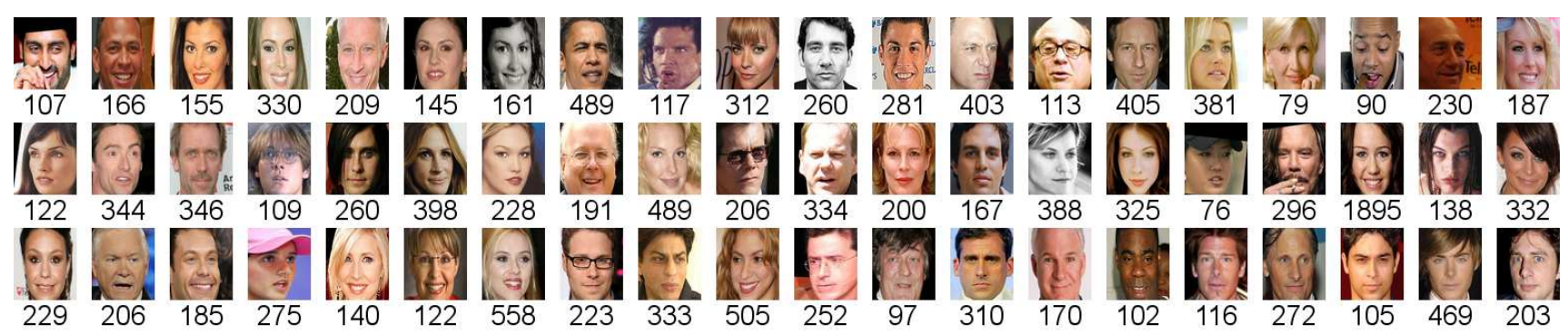

(a) PubFig Development set (60 individuals)

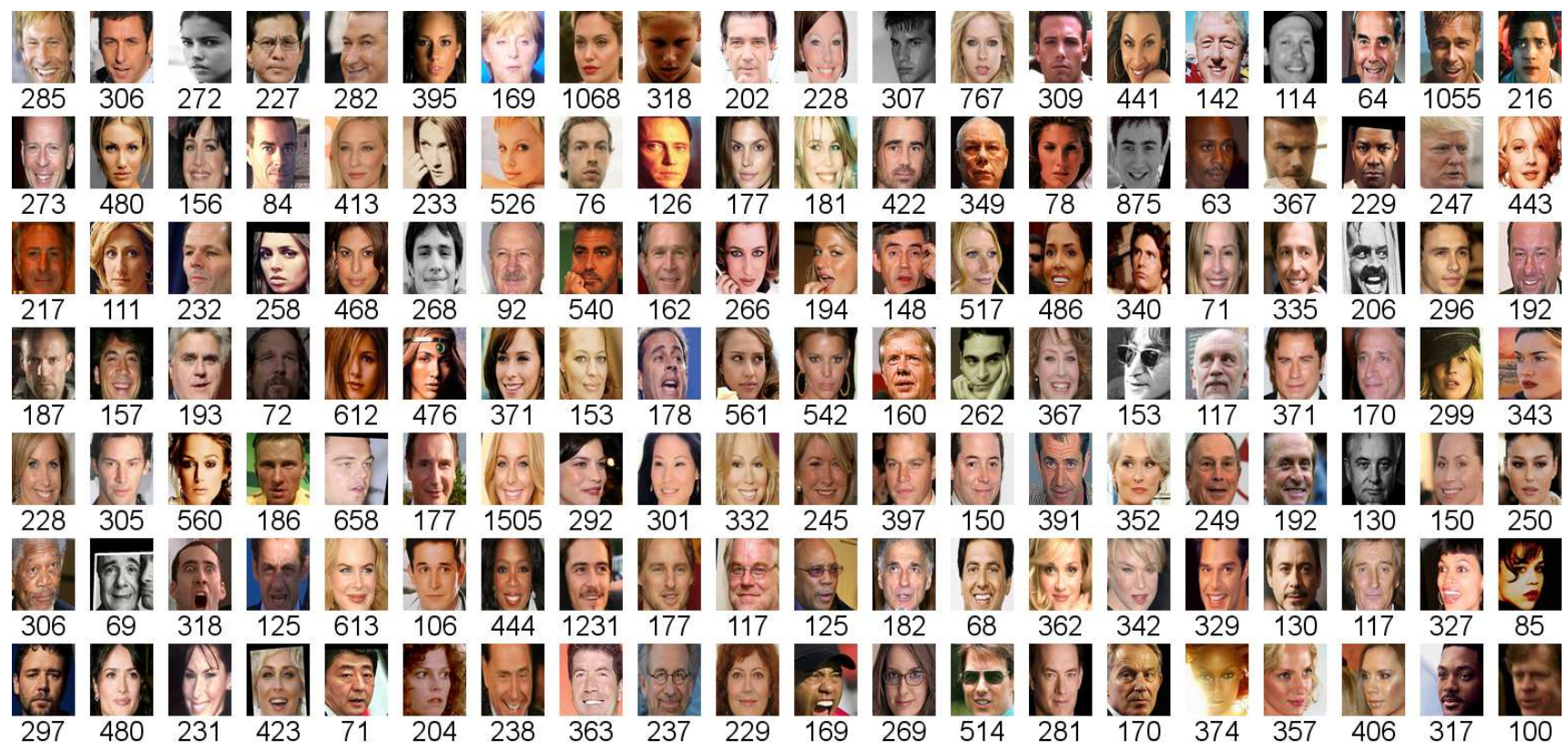

(b) PubFig Evaluation set (140 individuals)

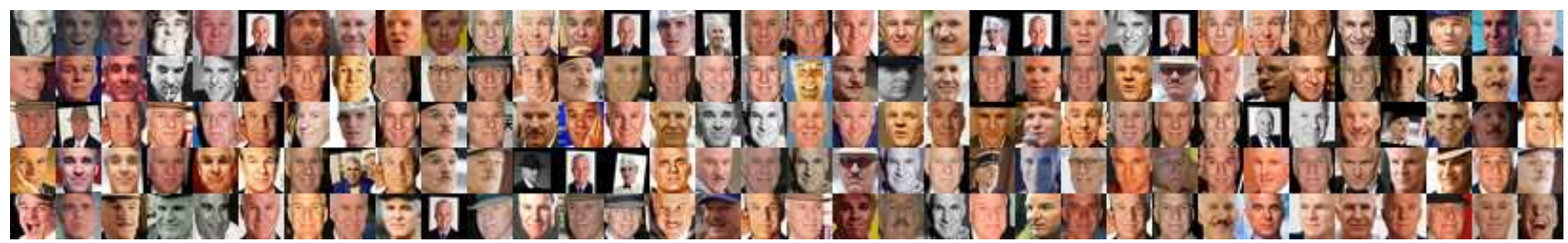

(c) All 170 images of Steve Martin

Fig. 4. The PubFig dataset consists of 58,797 images of 200 public figures - celebrities and politicians - partitioned into (a) a development set of 60 individuals and (b) an evaluation set of 140 individuals. Below each thumbnail is shown the number of photos of that person. There is no overlap in either identity or image between the development set and any dataset that we evaluate on, including Labeled Faces in the Wild (LFW) [27]. The immense variability in appearance captured by PubFig can be seen in (c), which shows all 170 images of Steve Martin.

from news sources, and exhibit many of the same types of variation as the Columbia Face Dataset.

\subsection{Collecting Attribute and Identity Labels}

For labeling images in our Columbia Face Database, we use the Amazon Mechanical Turk (MTurk) service. This service matches workers to online jobs created by requesters, who can optionally set quality controls such as requiring confirmation of results by multiple workers, filters on minimum worker experience, etc.

We submitted 110, 000 attribute labeling jobs showing 30 images to 3 workers per job, presenting a total of over 10 million images to users. The jobs asked workers to select face images which exhibited a specified attribute. (A few manually-labeled images were shown as examples.) Only labels where all 3 people agreed were used. From this raw data, we were able to collect over 145,000 triply-verified positive attribute labels, for about $\$ 6,000$.

Although this approach is somewhat similar to other labeling efforts in the computer vision community - such as ImageNet [15] and LabelMe [48], which focus on naming objects, images, and regions of images using nouns - there are several important 


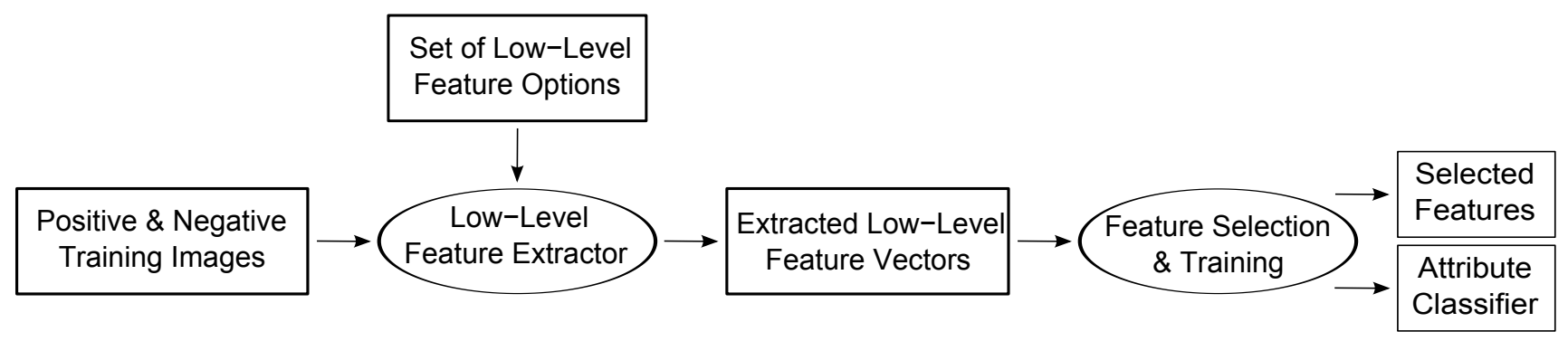

Fig. 5. Overview of attribute training architecture. Given a set of labeled positive and negative training images, low-level feature vectors are extracted using a large pool of low-level feature options. Each feature option consists of a region chosen from Fig. 6 and a feature type chosen from Table 1. An automatic, iterative selection process then picks the best set of features for correctly classifying the input data. The outputs are the selected features and the trained attribute classifier.

differences. One is that attributes need not be binary or even discrete; a person's age or the thickness of their eyebrows are both continuous attributes. (However, in this work we only consider discrete attributes, to simplify labeling.) Another critical difference is that visual attributes can be composed more freely than names, which generally exist in a tree-structured hierarchy. This allows for the use of a set of general attributes, which can be combined in an exponential number of ways to describe many objects at different levels of specificity. Attributes can therefore compactly provide a great deal of information, both about object properties and their identity. Finally, for many objects, it can be prohibitively expensive to obtain a large number of labeled training images of a specific object or category. In contrast, the same attribute can be exhibited by many otherwise-unrelated objects, making it easier to find more training images.

For gathering identity labels, we used the images downloaded from keyword searches on people's names as raw inputs, which were then filtered to create the final set. We submitted MTurk jobs asking users to select only the face images of a given person (of whom a few examples were shown). We also ran additional jobs pruning images for quality, good alignment, and some conservative duplicate-removal.

From these attribute and identity labels and our face database, we have created two publicly available face datasets, described next.

\subsection{FaceTracer Dataset}

The FaceTracer dataset is a subset of the Columbia Face Database, and it includes attribute labels. Each of the 15,000 faces in the dataset has a variety of metadata and fiducial points marked. The attributes labeled include demographic information such as age and race, facial features like mustaches and hair color, and other attributes such as expression, environment, etc. There are 5,000 labels in all. FaceTracer can be used as simply a dataset of real-world images with face detections and fiducials; or by researchers wanting to train their own attribute classifiers; or for any other non-commercial purpose.
The dataset is publicly available as a set of face URLs and accompanying data at http://faceserv.cs. columbia.edu/databases/facetracer/

\subsection{PubFig Dataset}

The PubFig dataset is a more direct complement to the LFW dataset [27]. It consists of 58,797 images of 200 public figures. The larger number of images per person (as compared to LFW) allows one to construct subsets of the data across different poses, lighting conditions, and expressions for further study. Figure $4 \mathrm{c}$ shows the variation present in all the images of a single individual. In addition, this dataset is wellsuited for recognition experiments.

PubFig is divided into a development set of 60 people (shown in Fig. 4a), on which we trained our simile classifiers (described in Sec. 4.4), and an evaluation set of 140 people (shown in Fig. 4b). The evaluation set was used to create a face verification benchmark similar to that from LFW.

All the data (with URLs to images) and evaluation benchmarks from PubFig are publicly available for non-commercial use at http://faceserv.cs.columbia. edu/databases/pubfig/, which also includes information on pose, expression and illumination for the evaluation set, and the outputs of our attribute classifiers on all images in both the development and evaluation sets.

\section{Learning Visual Attributes}

Given a particular describable visual attribute - say "gender" - how can one train a classifier for the attribute? Let us first formalize our notion of attributes. Attributes can be thought of as functions $\mathbf{a}_{\mathbf{i}}$ that map images $I$ to real values $a_{i}$. Large positive values of $a_{i}$ indicate the presence or strength of the $i$ th attribute, while negative values indicate its absence.

Consider the attribute "gender." If images $I_{1}$ and $I_{2}$ are of males and image $J$ is of a female, the gender function $\mathbf{a}_{\mathrm{g}}$ should map the males to positive values and $J$ to a negative value, even if $I_{1}$ and $I_{2}$ differ in other respects such as lighting, pose, age, expression, 


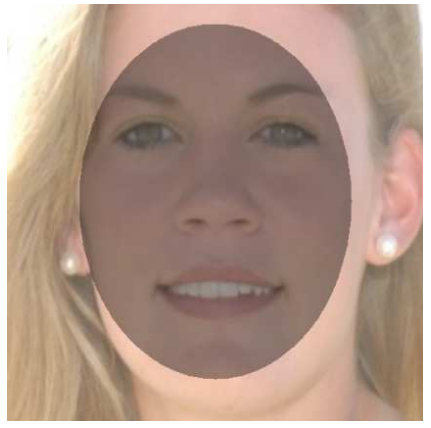

(a)

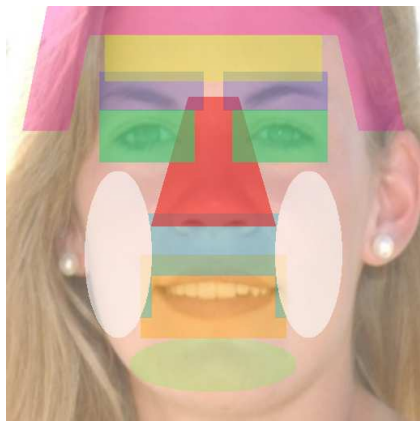

(b)
Fig. 6. The face regions used for automatic feature selection are shown here on an affine-aligned face image. There is (a) one region for the whole face, and (b) nine regions corresponding to functional parts of the face, such as the mouth, eyes, nose, etc. Regions are large enough to contain the face part across changes in pose, small errors in alignment, and differences between individuals. The regions are manually defined, once, in the affine-aligned coordinate system, and can then be used automatically for all aligned input faces.

etc. The magnitudes of the outputs should measure the degree of the attribute. For instance, if $I_{1}$ were an image of Clint Eastwood and $I_{2}$ were an image of Orlando Bloom, we might want $\mathbf{a}_{\mathbf{g}}\left(I_{1}\right)>\mathbf{a}_{\mathbf{g}}\left(I_{2}\right)$.

Similes are another class of describable visual traits, which describe the similarity of a face region between two different individuals. For example, we could say a person has "eyes like Penelope Cruz's" or a "mouth like Angelina Jolie's." We can formalize these two

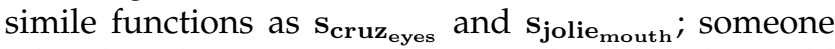
who shared Cruz's eyes but not Jolie's mouth would thus have a positive value for the former and a negative value for the latter.

Learning an attribute or simile classifier consists of fitting a function to a set of labeled training data. If the training labels are \pm 1 , this can be seen as fitting a classification function; real-valued labels imply regression; and if only ordering constraints are given, it becomes a problem of learning ranking functions. In all cases, regularization is important because the inputs (low-level image features) are very high-dimensional with complex variation, and there is always limited training data. This regularization could be biased by the distribution of features actually observed, which can be acquired from both labeled and unlabeled data. In this work, we consider mainly binary classifiers; regressors would likely behave very similarly, though possibly with greater accuracy.

\subsection{Training Architecture}

An overview of the attribute training architecture is shown in Fig. 5. The key idea is to leverage the many efficient and effective low-level features that have been developed by the computer vision community, choosing amongst a large set of them to find the ones suited for learning a particular attribute. This process
TABLE 1

Feature type options. A complete feature type is constructed by first converting the pixels in a given region (see Fig. 6) to one of the pixel value types from the first column, then applying one of the normalizations from the second column, and finally aggregating these values into the output feature vector using one of the options from the last column.

\begin{tabular}{|c|c|c|}
\hline Pixel Value Types & Normalizations & Aggregation \\
\hline \hline RGB & None & None \\
HSV & Mean Normalization & Histogram \\
Image Intensity & Energy Normalization & Mean/Variance \\
Edge Magnitude & & \\
Edge Orientation & & \\
\hline
\end{tabular}

should ideally be done in a generic, application- and domain-independent way, but with the ability to take advantage of domain-specific knowledge where available.

For the domain of faces, this knowledge consists of an affine alignment procedure and the use of lowlevel features which have proven to be very useful in a number of leading vision techniques, especially for faces. The alignment takes advantage of the fact that all faces have common structure - i.e., two eyes, a nose, a mouth, etc.- and that we have fiducial point detections available from a face detector [39]. The lowlevel features are described next.

\subsection{Low-Level Features}

As described in Sec. 3.1, face images are first aligned using an affine transformation. A set of $k$ low-level feature extractors $\mathbf{f}_{\mathbf{j}}$ are applied to an aligned input image $I$ to form a feature set $\mathcal{F}(I)$ :

$$
\mathcal{F}(I)=\left\{\mathbf{f}_{\mathbf{1}}(I), \cdots, \mathbf{f}_{\mathbf{k}}(I)\right\} .
$$

We describe each extractor $\mathbf{f}_{\mathbf{j}}$ in terms of four choices: the region of the face to extract features from, the type of pixel data to use, the kind of normalization to apply to the data, and finally, the level of aggregation to use.

The complete set of our 10 regions are shown in Fig. 6. The regions correspond to functional parts of a face, such as the nose, mouth, etc., similar to those defined in the work on modular eigenspaces [42]. Regions are defined manually in the affine-aligned coordinate system. This only has to be done once, after which all aligned faces can use the same region definitions. Our coarse division of the face allows us to take advantage of the common geometry shared by faces, while allowing for differences between individual faces as well as robustness to small errors in alignment. Prior to feature extraction, we mask out the background to avoid contaminating the classifiers. We also use the detected yaw angles of the face to first flip images so that they always face left. This small tweak makes the classifier's job slightly easier, as the 
"good" side of the face is always on the same half of the image.

From each region, one can extract different types of information, as categorized in Table 1. The types of pixel data to extract include various color spaces (RGB, HSV) as well as edge magnitudes and orientations. To remove lighting effects and better generalize across a limited number of training images, one can optionally normalize these extracted values. One method for normalization is mean normalization, $\hat{x}=\frac{x}{\mu}$, which removes illumination gains. Another option is energy normalization, $\hat{x}=\frac{x-\mu}{\sigma}$, which removes gains as well as offsets. (In these equations, $x$ refers to the input value, $\mu$ and $\sigma$ are the mean and standard deviation of all the $x$ values within the region, and $\hat{x}$ refers to the normalized output value.) Finally, one can aggregate normalized values over the region rather than simply concatenating them. This can be as simple as using only the mean and variance, or include more information by computing a histogram of values over the region. A complete feature type is created by choosing a region from Fig. 6 and one entry from each column of Table 1. (Of course, not all possible combinations are valid; e.g., it doesn't make sense to normalize hues.)

\subsection{Attribute Classifiers}

In creating a classifier for a particular attribute, we could simply extract all types of low-level features from the whole face, and let a classifier figure out which are important for the task and which are not. This, however, puts too great a burden on the classifier, confusing it with non-discriminative features. Instead, we design a selection procedure which automatically chooses the best features from a rich set of feature options. The chosen features are used to train the final attribute or simile classifier.

Attribute classifiers $C_{i}$ are built using a supervised learning approach. Training requires a set of labeled positive and negative images for each attribute, examples of which are shown in Fig. 7. The goal is to build a classifier that best classifies this training data by choosing an appropriate subset of the feature set $\mathcal{F}(I)$ described in the previous section. We do this iteratively using forward feature selection. In each iteration, we first train several individual classifiers on the current set of features in the output set, concatenated with a single region-feature combination. Each classifier's performance is evaluated using crossvalidation. The features used in the classifier with the highest cross-validation accuracy are added to the output set. We continue adding features until the accuracy stops improving, up to a maximum of 6 lowlevel features. For computational reasons, we drop the lowest-scoring $70 \%$ of features at each round, but always keeping at least 10 features.

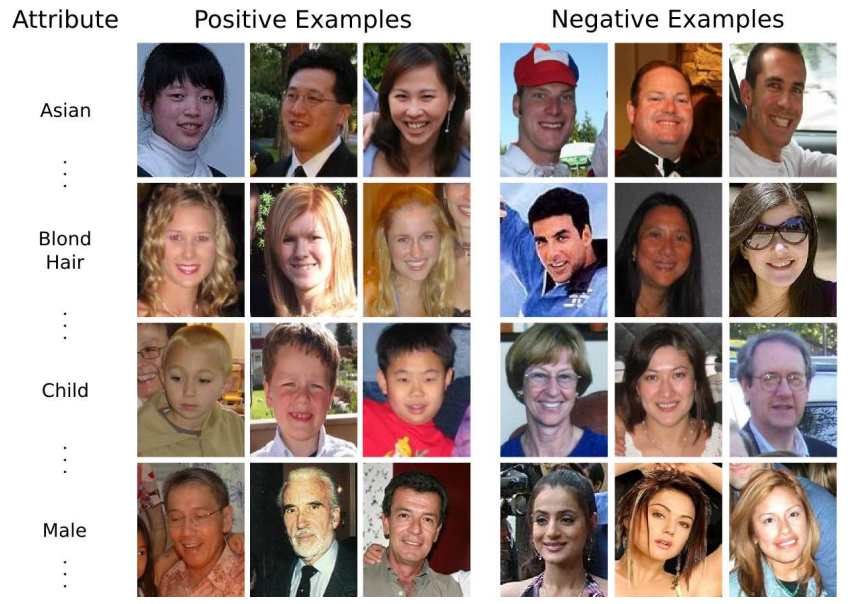

Fig. 7. Training data for the attribute classifiers consists of face images that match the given attribute label (positive examples) and those that don't (negative examples). Shown here are a few of the training images used for four different attributes. Final classifier accuracies for all 73 attributes are shown in Table 3.

Our classifiers are Support Vector Machines (SVMs) [11] with RBF kernels, trained using libsvm [8]. For each classifier, we use between 500 to 2000 positive and negative examples each, and perform a grid search over the $C$ and $\gamma$ parameters. The entire process is fully automatic, and takes a few hours of computation time per attribute trained, using a small grid of roughly 10 Intel Xeon processors, running at 3.0 Ghz each. We note that our procedure is by no means optimal; picking optimal features for non-linear classifiers is still an open problem in machine learning. Nevertheless, we obtain excellent results in practice.

While we have designed our classifier architecture to be flexible enough to handle a large variety of attributes, it is important to ensure that we have not sacrificed accuracy in the process. We therefore compare our approach to three previous state-of-theart methods for attribute classification: full-face SVMs using brightness normalized pixel values [35], Adaboost using Haar-like features [50], and Adaboost using pixel comparison features [1]. Since these works have mostly focused on gender classification, we use that attribute as the first testing criteria. In addition, we also test performance on the "smiling" attribute which we expect to be localizable to a small region of the face: the mouth.

Results are shown in Table 2. Our method performs the best in all cases (in some cases significantly so). This highlights the power of doing feature selection; in particular, we see that the full-face SVM method, while performing reasonably well on gender, did much worse on a localized attribute like smiling. Note that for the purposes of this test, we limited training and evaluation images to mostly frontal faces.

Using the Columbia Face Database and the learning 
TABLE 2

Comparison of attribute classification performance for "gender" and "smiling" attributes. Our fully-automatic feature selection and training procedure learns better classifiers than prior state-of-the-art methods for both attributes. Note that for this comparison, classifiers were trained and evaluated using only near-frontal faces.

\begin{tabular}{l|cc} 
Classification Method & $\begin{array}{c}\text { Gender } \\
\text { Error Rate }\end{array}$ & $\begin{array}{c}\text { Smiling } \\
\text { Error Rate }\end{array}$ \\
\hline Attribute Classifiers & $\mathbf{8 . 6 2} \%$ & $\mathbf{4 . 6 7 \%}$ \\
Pixel comp. feats. [1] & $13.13 \%$ & $7.41 \%$ \\
Haar-like feats. [50] & $12.88 \%$ & $6.40 \%$ \\
Full-face SVM [35] & $9.52 \%$ & $13.54 \%$
\end{tabular}

TABLE 3

Cross-validation accuracies of our 73 attribute classifiers.

\begin{tabular}{lc|lc} 
Attribute & Acc. & Attribute & Acc. \\
\hline Gender & $85.8 \%$ & Nose Size & $86.5 \%$ \\
Asian & $93.8 \%$ & Nose Shape & $87.0 \%$ \\
Caucasian & $91.5 \%$ & Nose-Mouth Lines & $93.2 \%$ \\
African American & $94.6 \%$ & Mustache & $92.5 \%$ \\
Indian & $91.9 \%$ & Mouth Closed & $90.0 \%$ \\
Baby & $93.0 \%$ & Mouth Open & $84.6 \%$ \\
Child & $80.3 \%$ & Mouth Wide Open & $89.0 \%$ \\
Youth & $87.7 \%$ & Lip Thickness & $82.4 \%$ \\
Middle-Aged & $84.9 \%$ & Wearing Lipstick & $86.7 \%$ \\
Senior & $92.0 \%$ & Teeth Visible & $91.2 \%$ \\
Black Hair & $90.8 \%$ & 5 o'clock Shadow & $89.3 \%$ \\
Blond Hair & $88.4 \%$ & Beard & $88.7 \%$ \\
Brown Hair & $74.9 \%$ & Goatee & $80.4 \%$ \\
Gray Hair & $89.9 \%$ & Double Chin & $81.0 \%$ \\
Bald & $90.4 \%$ & Jaw Shape & $66.1 \%$ \\
Wearing Hat & $89.1 \%$ & Chubby Face & $81.2 \%$ \\
Curly Hair & $70.1 \%$ & Oval Face & $73.3 \%$ \\
Wavy Hair & $66.6 \%$ & Square Face & $78.6 \%$ \\
Straight Hair & $78.4 \%$ & Round Face & $75.5 \%$ \\
Receding Hairline & $86.8 \%$ & Heavy Makeup & $89.0 \%$ \\
Bangs & $91.5 \%$ & Shiny Skin & $84.2 \%$ \\
Visible Forehead & $89.3 \%$ & Pale Skin & $89.4 \%$ \\
Obscured Forehead & $77.0 \%$ & Flushed Face & $88.8 \%$ \\
Blocked Forehead & $81.2 \%$ & Smiling & $95.9 \%$ \\
Eyebrow Thickness & $94.6 \%$ & Frowning & $95.3 \%$ \\
Eyebrow Shape & $79.7 \%$ & Wearing Necktie & $83.7 \%$ \\
Eye Shape & $89.7 \%$ & Wearing Necklace & $67.3 \%$ \\
Eyes Open & $92.3 \%$ & Blurry Image & $93.4 \%$ \\
Eye Color & $86.8 \%$ & Harsh Lighting & $77.0 \%$ \\
No Eyewear & $93.3 \%$ & Flash Lighting & $73.4 \%$ \\
Eyeglasses & $92.4 \%$ & Soft Lighting & $68.5 \%$ \\
Sunglasses & $96.5 \%$ & Environment & $85.3 \%$ \\
Bags Under Eyes & $85.4 \%$ & Color Photo & $97.9 \%$ \\
Wearing Earrings & $77.6 \%$ & Posed Photo & $71.9 \%$ \\
Sideburns & $72.3 \%$ & Attractive Man & $74.2 \%$ \\
High Cheekbones & $86.1 \%$ & Attractive Woman & $82.6 \%$ \\
Rosy Cheeks & $86.2 \%$ & & \\
& & & \\
& & & \\
Bay & &
\end{tabular}

procedure just described, we have trained a total of 73 attribute classifiers. Their cross-validation accuracies are shown in Table 3, and typically range from $80 \%$ to $90 \%$. Analysis of the chosen features indicate that all regions and feature types are useful (to varying extents), suggesting the importance of performing feature selection.

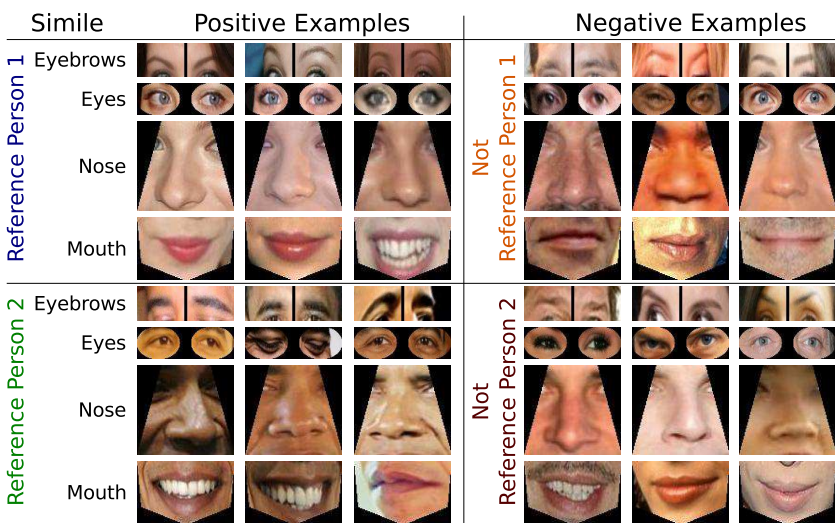

Fig. 8. Each simile classifier is trained using several images of a specific reference person, limited to a small face region such as the eyes, nose, or mouth. We show here three positive and three negative examples each, for four regions on two of the reference people used to train these classifiers.

\subsection{Simile Classifiers}

Simile classifiers measure the similarity of part of a person's face to the same part on a set of reference people. We use the 60 individuals from the development set of PubFig as the reference people. The left part of Fig. 8 shows examples of four regions selected from two reference people as positive examples. On the right are negative examples, which are simply the same region extracted from other individuals' images.

We emphasize two points. First, the individuals chosen as reference people do not appear in LFW or other benchmarks on which we produce results. Second, we train simile classifiers to recognize similarity to part of a reference person's face in many images, not similarity to a single image. The use of face parts increases the number of classifiers, but makes each one easier to learn, while the use of several input images allows for much better generalizability.

For each reference person, we train support vector machines to distinguish a region (e.g., eyebrows, eyes, nose, mouth) on their face from the same region on other faces. We manually choose eight regions and six feature types from the set of possible features described in Sec. 4.2 and train classifiers for each reference person/region/feature type combination, without feature selection, yielding 2,880 total simile classifiers. Each simile classifier is an RBF SVM, trained using at most 600 positive samples of a reference person and at most 10 times as many negative samples, randomly chosen from images of other people in the training set.

\section{Face Verification}

Existing methods for face verification - "are these two faces of the same person" - often make mistakes that would seem to be avoidable: men being confused for women, young people for old, asians for caucasians, 


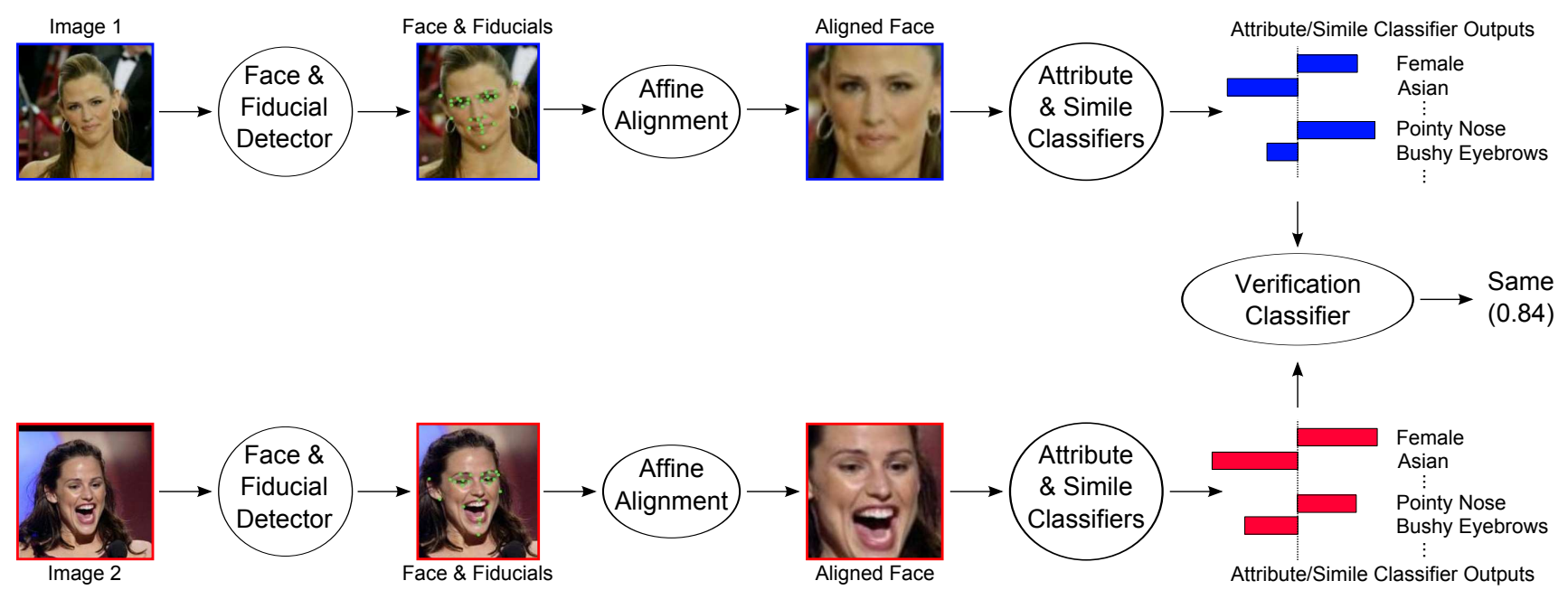

Fig. 9. The face verification pipeline. A pair of input images are run through a face and fiducial detector [39], and the fiducials are then used to align both faces to a canonical coordinate system. The aligned face images are fed to each of our attribute and simile classifiers individually to obtain a set of attribute values. Finally, these values are compared using a verification classifier to make the output determination, which is returned along with the distance to the decision boundary. The entire process is fully automatic.

etc. On the other hand, small changes in pose, expression, or lighting can cause two otherwise similar images of the same person to be misclassified by an algorithm as different. Based on this observation, we hypothesized that the attribute and simile classifiers could avoid such mistakes.

\subsection{Training a Verification Classifier}

Fig. 9 illustrates how attribute-based face verification is performed on a new pair of input images. In order to decide whether two face images $I_{1}$ and $I_{2}$ show the same person, one can train a verification classifier $V$ that compares attribute vectors $\mathbf{C}\left(I_{1}\right)$ and $\mathbf{C}\left(I_{2}\right)$ and returns $v\left(I_{1}, I_{2}\right)$, the verification decision. These vectors are constructed by concatenating the result of $n$ different attribute and/or simile classifiers.

To build $V$, let us make some observations about the particular form of our classifiers:

1) Values $C_{i}\left(I_{1}\right)$ and $C_{i}\left(I_{2}\right)$ from the $i$ th classifier should be similar if the images are of the same individual, and different otherwise.

2) Classifier values are raw outputs of binary classifiers, where the objective function is trying to separate examples around 0 . Thus, the signs of values should be important.

Let $a_{i}=C_{i}\left(I_{1}\right)$ and $b_{i}=C_{i}\left(I_{2}\right)$ be the outputs of the $i$ th trait classifier for each face $(1 \leq i \leq n)$. One would like to combine these values in such a way that our second-stage verification classifier $V$ can make sense of the data. This means creating values that are large (and positive) when the two inputs are of the same individual, and negative otherwise. For observation (1), we see that using the absolute difference $\left|a_{i}-b_{i}\right|$ will yield the desired outputs; for observation (2), the product $a_{i} b_{i}$. Putting both terms together yields the tuple $p_{i}$ :

$$
p_{i}=\left\langle\left|a_{i}-b_{i}\right|, a_{i} b_{i}\right\rangle
$$

The concatenation of these tuples for all $n$ attribute/simile classifier outputs forms the input to the verification classifier $V$ :

$$
v\left(I_{1}, I_{2}\right)=V\left(\left\langle p_{1}, \ldots, p_{n}\right\rangle\right)
$$

Training $V$ requires pairs of positive examples (two images of the same person) and negative examples (images of two different people). For the classification function, we use an SVM with an RBF kernel for $V$, trained using libsvm [8] with the default parameters of $C=1$ and $\gamma=1 /$ ndims, where ndims is the dimensionality of $\left\langle p_{1}, \ldots, p_{n}\right\rangle$.

\subsection{Experimental Setup}

We perform face verification experiments on the Labeled Faces in the Wild (LFW) benchmark [27] and also on our PubFig benchmark. For each computational experiment, a set of pairs of face images is presented for training, and a second set of pairs is presented for testing. In all experiments, not only are the images in the training and test sets disjoint, but there is also no overlap in the individuals used in the two sets. In addition, the individuals and images used to train the attribute and simile classifiers are disjoint from the testing sets.

\subsection{Attribute Classifier Results on LFW}

The LFW dataset consists of 13,233 images of 5,749 people, gathered from news photos, and organized into 2 "views":

1) A development set of 2,200 pairs for training and 1,000 pairs for testing, on which to build models and choose features; and 
2) A 10-fold cross-validation set of 6,000 pairs, on which to evaluate final performance.

We used View 1 for high-level model selection (e.g., representation for the final classifier $V$ ) and evaluated our performance on each of the folds in View 2 using the "image restricted configuration," as described in the LFW paper [27].

A verification classifier $V$ is trained using nine folds from View 2 of LFW and then evaluated on the remaining fold, cycling through all ten folds. Receiver Operating Characteristic (ROC) curves are obtained by saving the classifier outputs for each test pair in all ten folds and then sliding a threshold over all output values to obtain different false positive/detection rates. An overall accuracy is obtained by using only the signs of the outputs (e.g., thresholding at 0 ) and counting the number of errors in classification. The standard deviation for the accuracy is obtained by looking at the accuracies for each fold individually.

Fig. 10 shows results on LFW for our attribute classifiers (red line), simile classifiers (blue line), and a hybrid of the two (green line), along with several previous methods (dotted lines) [26], [47], [55], [56], [59], [60]. The accuracies for each of our methods are $85.25 \% \pm 1.58 \%, 84.14 \% \pm 1.31 \%$, and $85.54 \% \pm 1.23 \%$, respectively. ${ }^{2}$ Our highest accuracy of $85.54 \%$ is comparable to the $86.83 \%$ accuracy of the current stateof-the-art method [60] on LFW. The small bump in performance from combining the attribute and simile classifiers suggests that while they contain much of the same kind of information, there are still some interesting differences. This can be better seen in Fig. 10, where similes do better in the low-falsepositive regime, but attributes do better in the highdetection-rate regime.

\subsection{Human Attribute Labels on LFW}

Although our methods already achieve close to the current best performance on LFW, it is interesting to consider how well attribute classifiers could potentially do. There are several reasons to believe that our results are only first steps towards this ultimate goal:

- We have currently trained 73 attribute classifiers. Adding more attributes, especially fine-scale ones such as the presence and location of highly discriminative facial features including moles, scars, and tattoos, should greatly improve performance.

- Of the 73 attributes, many are not discriminative for verification. For example, facial expression, scene illumination, and image quality are all unlikely to aid in verification. There is also a severe imbalance in LFW of many basic attributes such as gender and age, which reduces the expected benefit of using these attributes for verification.

2. Our face detector was unable to detect one or more faces in 53 of the 6,000 total pairs. For these, we assumed average performance.

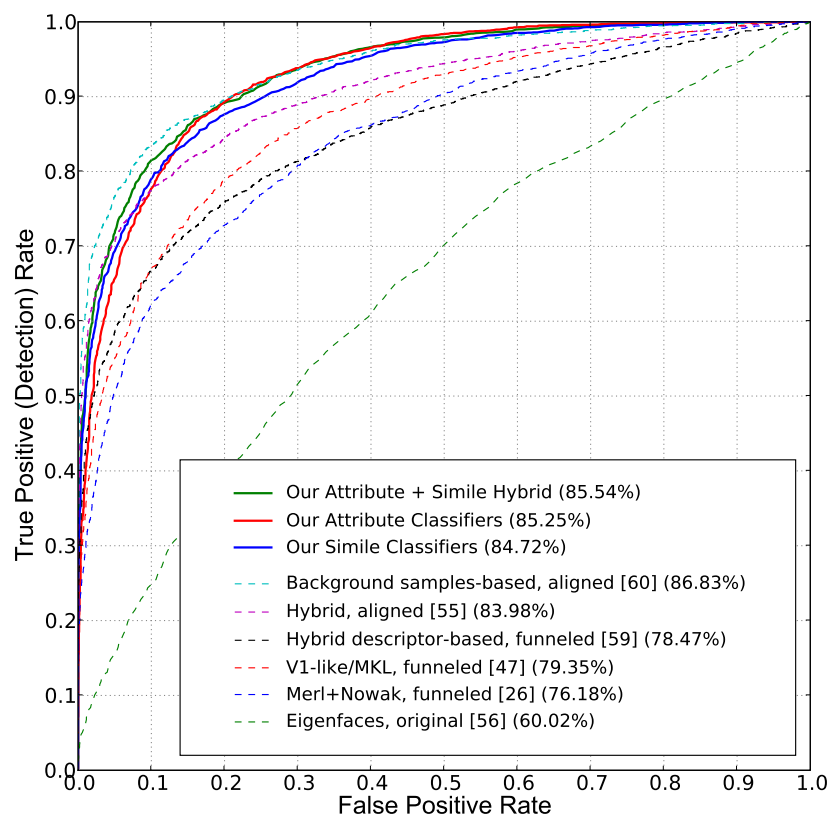

Fig. 10. Face verification performance on LFW of our attribute classifiers, simile classifiers, and a hybrid of the two are shown in solid red, blue, and green, respectively. Dashed lines are existing methods. Our highest accuracy is $85.54 \%$, which is comparable to the current state-of-the-art accuracy of $86.83 \%$ [60]. Notice that similes perform better at low false positive rates, attributes better at high detection rates, and hybrid better throughout.

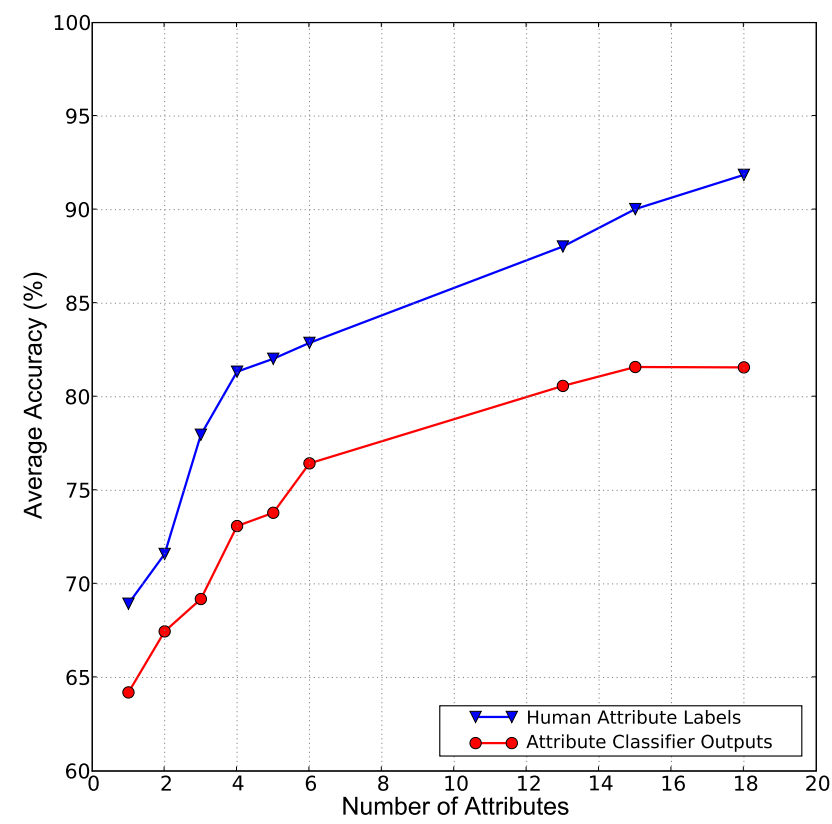

Fig. 11. Comparison of face verification performance on LFW using human attribute labels (blue line) vs. automaticallycomputated classifier outputs (red line). Verification using human labels consistently outperforms that using classifier outputs. With 18 attributes, human attribute labels reach $91.86 \%$ accuracy, compared to only $81.57 \%$ using classifier outputs. Training better attribute classifiers (or regressors) could thus greatly improve verification performance. 
- The attribute functions were trained as binary classifiers rather than as continuous regressors. While we use the distance to the separationboundary as a measure of degree of the attribute, using regression may improve results.

With the hope of exploring what might be possible given better attribute classifiers, we performed an experiment in which our automatic attribute labeling process was replaced by human labels, keeping the verification process identical. MTurk workers were asked to label attributes for all faces in the LFW View 2 benchmark set. We averaged seven user-responses per image to obtain smoothed estimates of the attribute values.

Fig. 11 shows a comparison of face verification performance on LFW using either these human attribute labels (blue line) or our automatically-computed classifier outputs (red line), for increasing numbers of attributes. In both cases, the labels are fed to the verification classifier $V$ and training proceeds identically, as described earlier. The set of attributes used for each corresponding point on the graphs were chosen manually (and identical for both). Verification results using the human attribute labels reach $91.86 \%$ accuracy with 18 attributes, significantly outperforming our computed labels at $81.57 \%$ for the same 18 attributes. Moreover, the drop in error rates from computational to human labels is actually increasing with more attributes, suggesting that adding more attributes could further improve accuracies.

\subsection{Human Verification on LFW}

The high accuracies obtained in the previous section lead to a natural question: How well do people perform on the verification task itself? While many algorithms for automatic face verification have been designed and evaluated on LFW, there are no published results about how well people perform on this benchmark. To this end, we conducted several experiments on human verification.

We followed the procedure of O'Toole et al. [40] to obtain this data, using Amazon Mechanical Turk. MTurk users were shown pairs of faces from the LFW View 2 benchmark set and asked to mark whether the images showed the same person or not. This was done on a scale of -1 to +1 , where the sign of the score was their decision, and the magnitude was their confidence in their response. The responses of 10 different users were averaged per face pair to get a score for that pair. (Thus, for the 6,000 image pairs in LFW, we gathered 60,000 data points from users for each of the three tests described below, for a total of 240,000 user inputs.) An ROC curve was created by sliding the confidence threshold from -1 to +1 , counting scores less than the threshold as "different" and those above as "same."

Results are shown in Fig. 12. Using the original LFW images (red curve), people have $99.20 \%$ accuracy

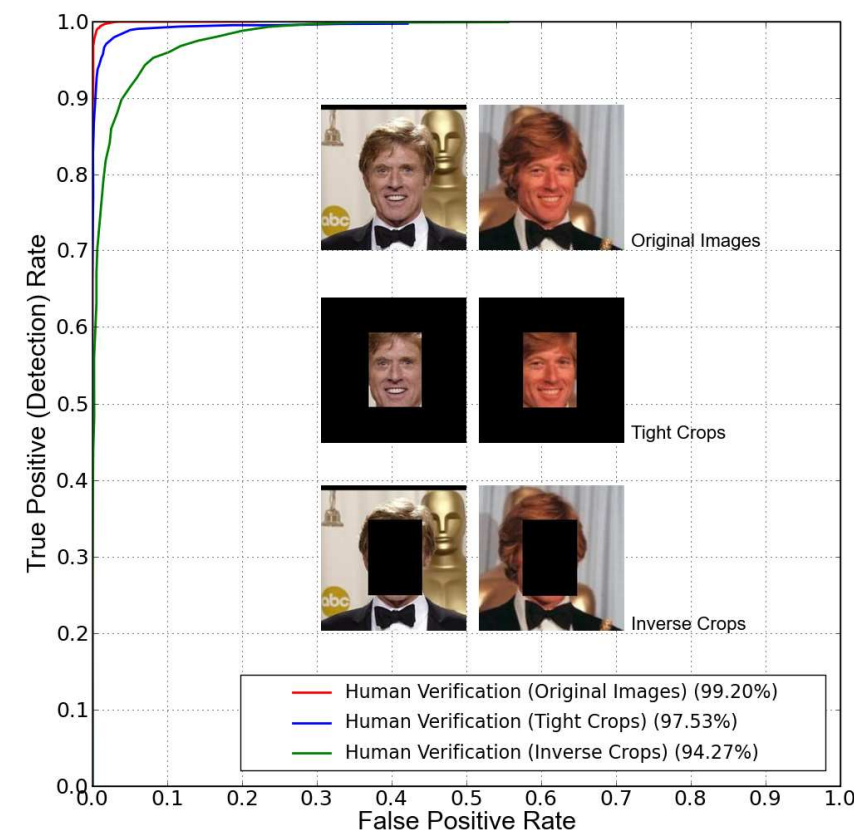

Fig. 12. Face verification performance on LFW by humans is almost perfect $(99.20 \%)$ when people are shown the original images (red line). Showing a tighter cropped version of the images (blue line) drops their accuracy to $97.53 \%$, due to the lack of available context. The green line shows that even with an inverse crop, i.e., when only the context is shown, humans still perform quite well, at $94.27 \%$. This highlights the strong context cues available on the LFW dataset.

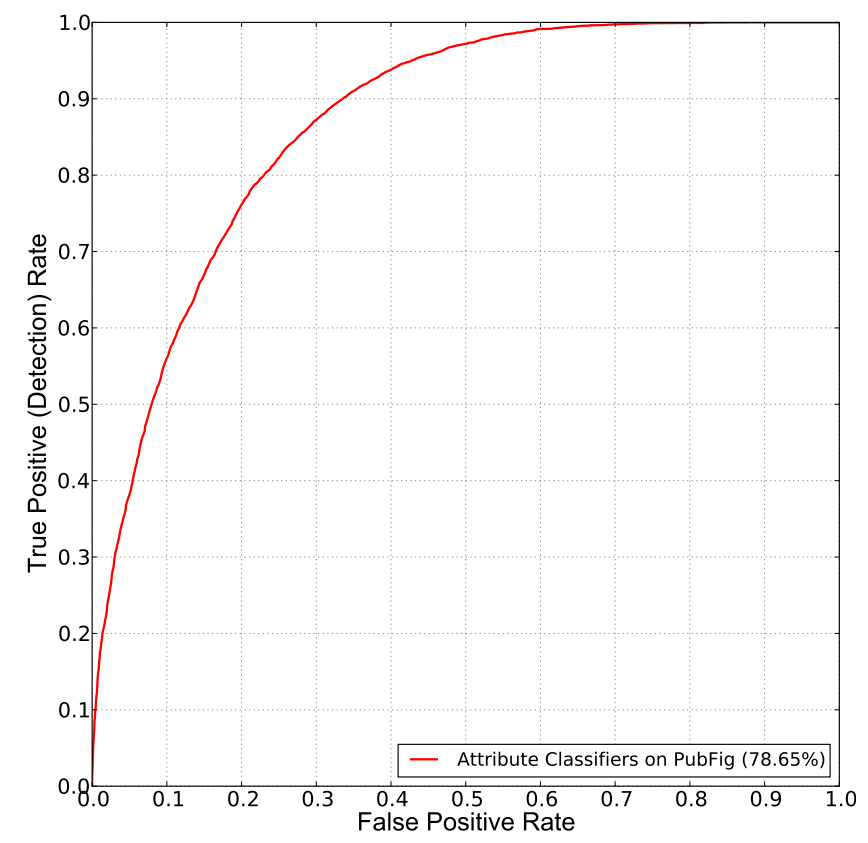

Fig. 13. Face verification results on the PubFig evaluation benchmark using our attribute classifiers. Our accuracy is $78.65 \%$ on this benchmark, which consists of 20,000 face pairs partitioned into 10 folds for cross-validation. Our lower performance on this experiment as compared to LFW suggests that it is a more challenging dataset. 


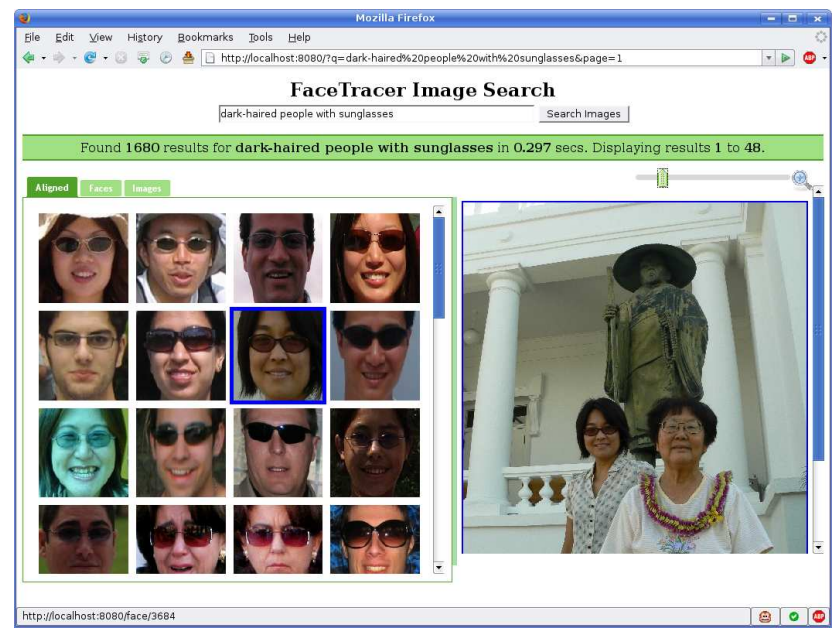

(a) Results for "dark-haired people with sunglasses"

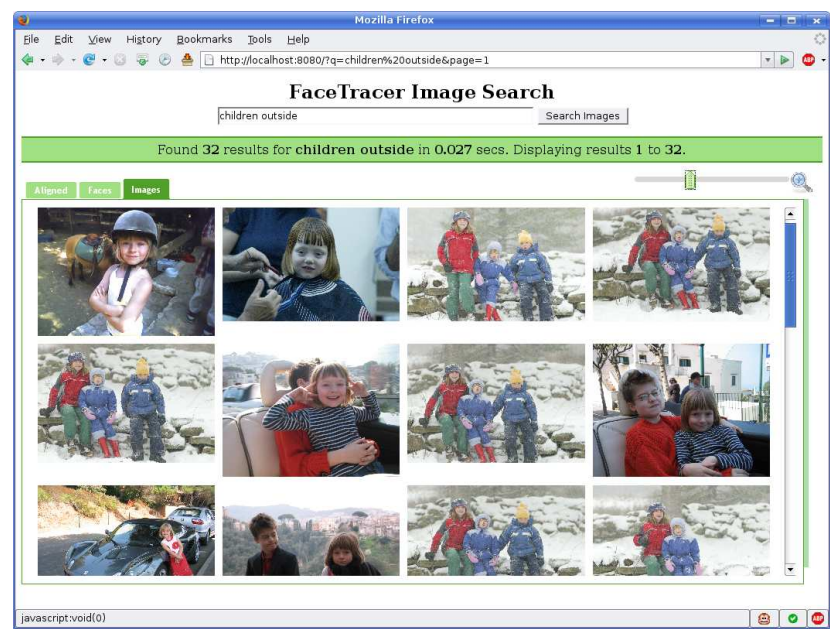

(b) Personalized results for "children outside"

Fig. 14. Results of queries (a) "dark-haired people with sunglasses" and (b) "children outside," using our attribute-based face search engine. In (a), search results are shown in the left panel as cropped faces, while the right panel shows a preview of the original image for the selected face. Clicking the image takes the user to the image's original webpage. (b) shows search results on a personalized dataset constructed from a single user's photos, displayed as thumbnails of the original images. In both cases, only relevant results are found. Also, note that the results in (b) were correctly classified as being "outside" using only the cropped face images, showing that faces often contain enough information to describe properties of the image not directly related to faces.

- essentially perfect. We then made the task tougher by cropping the images, leaving only the face visible (including at least the eyes, nose and mouth, and possibly parts of the hair, ears, and neck). This experiment measures how much people are helped by the context (sports shot, interview, press conference, etc.), background (some images of individuals were taken with the same background), and hair (although sometimes it is partially visible). The results (blue curve) show that performance drops to $97.53 \%$ - a tripling of the error rate.

To confirm that the region outside of the face is indeed helping people with identification, we ran a third experiment where the mask was inverted, i.e., we blacked out the face but showed the remaining part of the image. Surprisingly, people still achieve $94.27 \%$ accuracy, as shown by the green line in Fig. 12. These results reinforce the results of Sinha et al. [52], that context and hair are powerful cues for face recognition. It also perhaps points to a bias in LFW many news photos tend to be taken at the same event, making the face recognition task easier.

\subsection{Attribute Classifier Results on PubFig}

The PubFig benchmark, being much deeper (more images per person) and gathered from more varied sources, should ameliorate this issue. We test this hypothesis using an evaluation benchmark similar to LFW's. Face verification is performed on 20,000 pairs of images of 140 people, divided into 10 crossvalidation folds with mutually disjoint sets of 14 people each. These people are separate from the 60 people in the development set of PubFig, which were used for training the simile classifiers. The performance of our attribute classifiers on this benchmark is shown in Fig. 13, and it is indeed much lower than on LFW, with an accuracy of $78.65 \%$.

\section{Face Search}

Image search engines are currently dependent on textual metadata. This data can be in the form of filenames, manual annotations, or surrounding text. However, for the vast majority of images on the internet (and in peoples' private collections), this data is often ambiguous, incorrect, or simply not present. This presents a great opportunity to use attribute classifiers on images with faces, thereby making them searchable. To facilitate fast searches on a large collection of images, all images are labeled in an offline process using attribute classifiers. The resulting attribute labels are stored for fast online searches using the FaceTracer engine [29].

The FaceTracer engine uses simple text-based queries as inputs, since these are both familiar and accessible to most internet users, and correspond well to describable visual attributes. Search queries are mapped onto attribute labels using a dictionary of terms. Users can see the list of attributes supported by the system on the search page, allowing them to construct searches without having to guess what kinds of queries are allowed. This approach is simple, flexible, and yields excellent results in practice. Furthermore, it is easy to add new phrases and attributes to the dictionary, or maintain separate dictionaries for searches in different languages.

Search results are ranked by confidence, so that the most relevant images are shown first. We use the 
computed distance to the classifier decision boundary as a measure of the confidence. For searches with multiple query terms, we combine the confidences of different attribute labels such that the final ranking shows images in decreasing order of relevance to all search terms. To prevent high confidences for one attribute from dominating the search results, we first convert the confidences into probabilities by fitting a held-out set of positive and negative examples to gaussian distributions, and then use the product of the probabilities as the sort criteria. This ensures that the images with high confidences for all attributes are shown first.

Example queries on our search engine are shown in Figs. 14a and 14b. The returned results are all highly relevant. Fig. 14b additionally demonstrates two other interesting things. First, it was run on a personalized dataset of images from a single user, showing that this method can be applied to specialized image collections as well as general ones. Second, it shows that we can learn useful things about an image using just the appearance of the faces within it - in this case determining whether the image was taken indoors or outdoors.

This attribute-based search engine can be used in many other applications, replacing or augmenting existing tools. In law enforcement, eyewitnesses to crimes could use this system to quickly narrow a list of possible suspects and then identify the actual criminal from the reduced list, saving time and increasing the chances of finding the right person. On the internet, our face search engine is a perfect match for social networking websites such as Facebook, which contain large numbers of images with people. Additionally, the community aspect of these websites would allow for collaborative creation of new attributes. Finally, people could use our system to more easily organize and manage their own personal photo collections. For example, searches for blurry or other poor-quality images can be used to find and remove all such images from the collection.

\section{Conclusions and Future Directions}

In this work, we have shown how to automatically train classifiers for describable aspects of visual appearance - attributes and similes. These classifiers are learned using large collections of labeled images obtained from the internet. We demonstrated the use of these describable attributes for performing face verification and image search. We showed performance comparable to or better than the state-of-the-art in all aspects of the work: attribute classification, face verification, and search (qualitatively). We have also made available two large and complementary datasets for use by the community to make further progress along these lines.

These seem to be promising first steps in a new direction, and there are many avenues to explore. The experiments with human attribute labeling in Sec. 5.4 suggest that adding more attributes and improving the attribute training process could yield great benefits for face verification. Another direction to explore is how best to combine attribute and simile classifiers with low-level image cues. Finally, an open question is how attributes can be applied to domains other than faces. It seems that for reliable and accurate attribute training, analogues to the detection and alignment process must be found.

\subsection{Dynamic Selection of Attributes to Label}

The set of attributes used in this work were chosen in an ad-hoc way; how to select them dynamically in a more principled manner is an interesting topic to consider. In particular, a system with a user-in-theloop could be used to suggest new attributes. Thanks to Amazon Mechanical Turk, such a system would be easy to setup and could operate autonomously.

The idea is to evaluate a current set of attribute classifiers on a verification dataset and look at the mistakes made by the algorithm. Presumably, these mistakes would occur on face pairs which could not be sufficiently distinguished using the current set of attributes. Borrowing terminology from color theory, we term these face pairs "metamers." The metamers could be shown to users on MTurk, asking them to suggest new attributes which could disambiguate such pairs. By doing this over a large enough number of images and users, one could grow an existing set of attributes in a maximally-efficient way. Measures based on mutual information and information gain could be used in association with this metamer disambiguation strategy to ensure that the best attributes were picked.

\section{ACKNOWLEDGMENTS}

The authors would like to thank Pietro Perona for suggesting the human attribute labels experiment described in Sec. 5.4. We are also grateful to the anonymous reviewers for their excellent suggestions. This work was supported in part by ONR award N00014-08-1-0638 and IARPA DA 911NF-10-2-0011.

\section{REFERENCES}

[1] S. Baluja and H. Rowley, "Boosting sex identification performance," IJCV, 2007.

[2] P. Belhumeur, J. Hespanha, and D. Kriegman, "Eigenfaces vs. Fisherfaces: Recognition using class specific linear projection," ECCV, pp. 45-58, 1996

[3] T. L. Berg, A. C. Berg, J. Edwards, M. Maire, R. White, Y.-W Teh, E. Learned-Miller, and D. Forsyth., "Names and faces in the news," CVPR, 2004.

[4] V. Blanz, S. Romdhani, and T. Vetter, "Face identification across different poses and illuminations with a $3 \mathrm{~d}$ morphable model," FGR, 2002.

[5] V. Bruce, Z. Henderson, K. Greenwood, P. J. B. Hancock, A. M. Burton, and P. I. Miller, "Verification of face identities from images captured on video," Journal of Experimental Psychology: Applied, vol. 5, pp. 339-360, 1999. 
[6] A. M. Burton, S. Wilson, M. Cowan, and V. Bruce, "Face recognition in poor-quality video: Evidence from security surveillance," Psychological Science, vol. 10, no. 3, pp. 243-248, 1999.

[7] C. D. Castillo and D. W. Jacobs, "Using stereo matching for 2-d face recognition across pose," CVPR, 2007.

[8] C.-C. Chang and C.-J. Lin, LIBSVM: a library for support vector

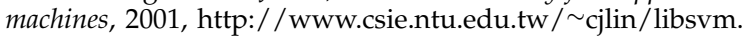

[9] H. Chen, P. Belhumeur, and D. Jacobs, "In search of illumination invariants," CVPR, 2000.

[10] T. Cootes, K. Walker, and C. Taylor, "View-based active appearance models," FGR, 2000.

[11] C. Cortes and V. Vapnik, "Support-vector networks," Machine Learning, vol. 20, no. 3, 1995

[12] G. W. Cottrell and J. Metcalfe, "Empath: face, emotion, and gender recognition using holons," in NIPS, 1990, pp. 564-571.

[13] N. Dalal and B. Triggs, "Histograms of oriented gradients for human detection," in IEEE Conference on Computer Vision and Pattern Recognition, vol. 1, 2005, pp. 886-893.

[14] R. Datta, J. Li, and J. Z. Wang, "Content-based image retrieval: Approaches and trends of the new age," Multimedia Information Retrieval, pp. 253-262, 2005.

[15] J. Deng, W. Dong, R. Socher, L.-J. Li, K. Li, and L. Fei-Fei, "ImageNet: A Large-Scale Hierarchical Image Database," in CVPR, 2009.

[16] M. Everingham, J. Sivic, and A. Zisserman, "Hello! my name is... Buffy - automatic naming of characters in TV video," $B M V C, 2006$

[17] M. Everingham, L. Van Gool, C. K. I. Williams, J. Winn, and A. Zisserman, "The PASCAL Visual Object Classes Challenge 2008 (VOC2008) Results," http://www.pascalnetwork.org/challenges/VOC/voc2008/workshop/index.htm

[18] A. Farhadi, I. Endres, D. Hoiem, and D. Forsyth, "Describing objects by their attributes," in CVPR, 2009.

[19] A. Ferencz, E. Learned-Miller, and J. Malik, "Learning to locate informative features for visual identification," IJCV Special Issue on Learning and Vision, 2007.

[20] V. Ferrari and A. Zisserman, "Learning visual attributes," in Advances in Neural Information Processing Systems, Dec. 2007.

[21] Y. Freund and R. Shapire, "Experiments with a new boosting algorithm," ICML, 1996.

[22] A. S. Georghiades, P. N. Belhumeur, and D. J. Kriegman, "From few to many: Illumination cone models for face recognition under variable lighting and pose," PAMI, vol. 23, no. 6, pp 643-660, 2001.

[23] B. A. Golomb, D. T. Lawrence, and T. J. Sejnowski, "SexNet A neural network identifies sex from human faces," in NIPS, 1990, pp. 572-577.

[24] R. Gross, J. Shi, and J. Cohn, "Quo vadis face recognition?" in Workshop on Empirical Evaluation Methods in Computer Vision, December 2001.

[25] G. Huang, V. Jain, and E. Learned-Miller, "Unsupervised joint alignment of complex images," ICCV, 2007.

[26] G. Huang, M. Jones, and E. Learned-Miller, "LFW results using a combined Nowak plus MERL recognizer," in Real-Life Images workshop at ECCV, 2008.

[27] G. Huang, M. Ramesh, T. Berg, and E. Learned-Miller, "Labeled Faces in the Wild: A database for studying face recognition in unconstrained environments," UMass Amherst Technical Report 07-49, October 2007.

[28] M. Kirby and L. Sirovich, "Application of the karhunen-loeve procedure for the characterization of human faces," Pattern Analysis and Machine Intelligence, IEEE Transactions on, vol. 12, no. 1 , pp. $103-108$, jan 1990.

[29] N. Kumar, P. N. Belhumeur, and S. K. Nayar, "FaceTracer: A search engine for large collections of images with faces," $E C C V, 2008$

[30] N. Kumar, A. C. Berg, P. N. Belhumeur, and S. K. Nayar, "Attribute and simile classifiers for face verification," ICCV 2009.

[31] C. Lampert, H. Nickisch, and S. Harmeling, "Learning to detect unseen object classes by between-class attribute transfer," in CVPR, 2009.

[32] M. S. Lew, N. Sebe, C. Djeraba, and R. Jain, "Content-based multimedia information retrieval: State of the art and challenges," ACM Trans. Multimedia Comput. Commun. Appl., vol. 2 no. 1 , pp. $1-19,2006$
[33] H. Ling, S. Soatto, N. Ramanathan, and D. Jacobs, "A study of face recognition as people age," ICCV, 2007.

[34] D. Lowe, "Distinctive image features from scale-invariant keypoints," IJCV, 2003.

[35] B. Moghaddam and M.-H. Yang, "Learning gender with support faces," TPAMI, vol. 24, no. 5, pp. 707-711, 2002.

[36] D. Nister and H. Stewenius, "Scalable recognition with a vocabulary tree," in CVPR, 2006, pp. 2161-2168.

[37] E. Nowak and F. Jurie, "Learning visual similarity measures for comparing never seen objects," CVPR, 2007.

[38] E. Nowak, F. Jurie, and B. Triggs, "Sampling strategies for bagof-features image classification," in In Proc. ECCV. Springer, 2006, pp. 490-503.

[39] Omron, "OKAO vision," http://www.omron.com/r_d/ coretech/vision/okao.html, 2009.

[40] A. O'Toole, P. Phillips, F. Jiang, J. Ayyad, N. Penard, and H. Abdi, "Face recognition algorithms surpass humans matching faces over changes in illumination," PAMI, vol. 29, no. 9, pp. 1642-1646, Sept. 2007.

[41] M. Palatucci, D. Pomerleau, G. Hinton, and T. Mitchell, "Zeroshot learning with semantic output codes," in NIPS, 2009.

[42] A. Pentland, B. Moghaddam, and T. Starner, "View-based and modular eigenspaces for face recognition," CVPR, pp. 84-91, 1994.

[43] A. Pentland, R. Picard, and S. Sclaroff, "Photobook: Contentbased manipulation of image databases," IJCV, pp. 233-254, 1996.

[44] P. Phillips, H. Moon, S. Rizvi, and P. Rauss, "The FERET evaluation methodology for face-recognition algorithms," PAMI, vol. 22, no. 10, pp. 1090-1104, 2000.

[45] P. J. Phillips, P. J. Flynn, T. Scruggs, K. W. Bowyer, J. Chang, K. Hoffman, J. Marques, J. Min, and W. Worek, "Overview of the face recognition grand challenge," CVPR, pp. 947-954 2005.

[46] P. Phillips, P. Flynn, T. Scruggs, K. Bowyer, and W. Worek, "Preliminary face recognition grand challenge results," FGR, pp. 15-24, 2006.

[47] N. Pinto, J. J. DiCarlo, and D. D. Cox, "How far can you get with a modern face recognition test set using only simple features?" in Computer Vision and Pattern Recognition, 2009.

[48] B. Russell, A. Torralba, and K. Murphy, "LabelMe: a database and web-based tool for image annotation," IJCV, vol. 77, no. 1-3, pp. 157-173, 2008.

[49] F. Samaria and A. Harter, "Parameterisation of a stochastic model for human face identification," Workshop on Applications of Computer Vision, 1994.

[50] G. Shakhnarovich, P. Viola, and B. Moghaddam, "A unified learning framework for real time face detection and classification," FGR, 2002.

[51] T. Sim, S. Baker, and M. Bsat, "The CMU pose, illumination, and expression (PIE) database," ICAFGR, pp. 46-51, 2002.

[52] P. Sinha, B. Balas, Y. Ostrovsky, and R. Russell, "Face recognition by humans: Nineteen results all computer vision researchers should know about," Proceedings of the IEEE, vol. 94 pp. 1948-1962, 2006.

[53] P. Sinha and T. Poggio, "I think i know that face..." Nature, vol. 384, no. 6608, p. 404, 1996.

[54] J. Sivic and A. Zisserman, "Video Google: A text retrieval approach to object matching in videos," in Proceedings of the International Conference on Computer Vision, vol. 2, Oct. 2003, pp. 1470-1477.

[55] Y. Taigman, L. Wolf, and T. Hassner, "Multiple one-shots for utilizing class label information," in The British Machine Vision Conference (BMVC), 2009.

[56] M. Turk and A. Pentland, "Face recognition using eigenfaces," CVPR, 1991

[57] P. Viola and M. Jones, "Rapid object detection using a boosted cascade of simple features," CVPR, 2001.

[58] L. Wiskott, J.-M. Fellous, N. Krüger, and C. von der Malsburg, "Face recognition by elastic bunch graph matching," IEEE Trans. Pattern Analysis and Machine Intelligence, vol. 19, pp. 775$779,1997$.

[59] L. Wolf, T. Hassner, and Y. Taigman, "Descriptor based methods in the wild," in Real-Life Images workshop at ECCV, 2008.

[60] - "Similarity scores based on background samples," in Asian Conference on Computer Vision, 2009. 
[61] W. Zhao, R. Chellappa, P. J. Phillips, and A. Rosenfeld, "Face recognition: A literature survey," ACM Comput. Surv., vol. 35 no. 4, pp. 399-458, 2003.

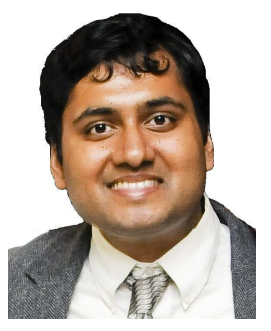

Neeraj Kumar received BSc degrees in Computer Science and Aeronautical Engineering from the Georgia Institute of Technology in 2005 (both with highest honors). He was awarded a three-year National Defense Science and Engineering Graduate (NDSEG) Fellowship by the American Society for Engineering Education in 2005. He is currently a Ph.D. candidate in Computer Science at Columbia University, where he is co-advised by Professors P.N. Belhumeur and S.K. Nayar. His main research interests are at the intersection of computer vision and machine learning - developing techniques for efficient search and recognition in large image databases.

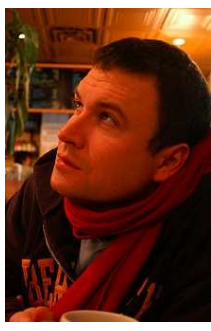

Alexander C. Berg received the $\mathrm{PhD}$ degree in Computer Science from U.C. Berkeley in 2005 . He is currently an assistant professor at Stony Brook University. Prior to that, Dr. Berg was a research scientist at Yahoo! Research and later Columbia University. His research addresses challenges in visual recognition at all levels, from image features, to high level semantics, with a focus on large scale vision problems and efficient computational solutions.

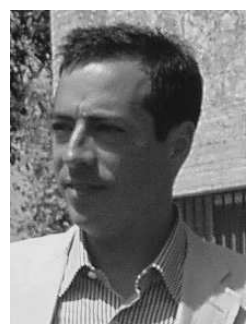

Peter N. Belhumeur received the ScB degree in Information Sciences from Brown University in 1985. He received the $\mathrm{PhD}$ degree in Engineering Sciences from Harvard University under the direction of David Mumford in 1993. He was a postdoctoral fellow at the University of Cambridge's Isaac Newton Institute for Mathematical Sciences in 1994. He was made Assistant, Associate, and Professor of Electrical Engineering at Yale University in 1994, 1998, and 2001, respectively. He joined Columbia University in 2002, where he is currently a Professor in the Department of Computer Science and the director of the Laboratory for the Study of Visual Appearance (VAP LAB). His main research focus is on illumination, reflectance, and shape, and their relation to visual appearance. Within these areas, he concentrates on two subproblems: the representation and recognition of objects under variable illumination and the estimation of the geometry of objects from low-level cues like image brightness, binocular stereopsis, and motion. Applications include face and object recognition, image-based rendering, computer graphics, content-based image and video compression, and human computer interfaces. He is a recipient of the Presidential Early Career Award for Scientists and Engineers (PECASE), the US National Science Foundation Career Award, and the Yale University Junior Faculty Fellowship. His papers have received the Siemens Best Paper Award at CVPR 1996, the Olympus Prize at ECCV 1998 and a Best Paper Honorable Mention Award at CVPR 2000.

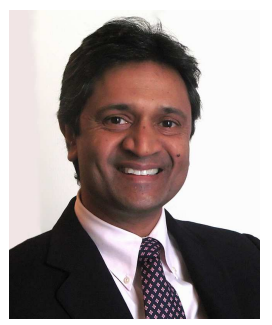

Shree K. Nayar received the $\mathrm{PhD}$ degree in Electrical and Computer Engineering from the Robotics Institute at Carnegie Mellon University in 1990. He is the T.C. Chang Professor of Computer Science at Columbia University and, since 2009, the chairman of the department. He heads the Columbia Vision Laboratory (CAVE), which is dedicated to the development of advanced computer vision systems. His research is focused on three areas: the creation of cameras that produce new forms of visual information, the modeling of the in teraction of light with materials, and the design of algorithms that recognize objects from images. His work is motivated by applications in the fields of computer graphics, human-machine interfaces, and robotics. Dr. Nayar has authored and coauthored papers that have received the Best Paper Award at the 2004 CVPR Conference held in Washington, DC, the Best Paper Honorable Mention Award at the 2000 IEEE CVPR Conference held in Hilton Head, the David Marr Prize at the 1995 ICCV held in Boston, the Siemens Outstanding Paper Award at the 1994 IEEE CVPR Conference held in Seattle, the 1994 Annual Pattern Recognition Award from the Pattern Recognition Society, the Best Industry Related Paper Award at the 1994 ICPR held in Jerusalem, and the David Marr Prize at the 1990 ICCV held in Osaka. He was the recipient of the Columbia Great Teacher Award in 2006, the Excellence in Engineering Teaching Award from the Keck Foundation in 1995, the NTT Distinguished Scientific Achievement Award from NTT Corporation, Japan, in 1994, the National Young Investigator Award from the US National Science Foundation in 1993, and the David and Lucile Packard Fellowship for Science and Engineering in 1992. In February 2008, he was elected to the National Academy of Engineering. 